\title{
Spatial effects, sampling errors, and task specialization in the honey bee
}

\author{
B. R. Johnson
}

Received: 14 August 2009/Revised: 23 January 2010/Accepted: 28 January 2010/Published online: 24 February 2010 (C) The Author(s) 2010. This article is published with open access at Springerlink.com

\begin{abstract}
Task allocation patterns should depend on the spatial distribution of work within the nest, variation in task demand, and the movement patterns of workers, however, relatively little research has focused on these topics. This study uses a spatially explicit agent based model to determine whether such factors alone can generate biases in task performance at the individual level in the honey bees, Apis mellifera. Specialization (bias in task performance) is shown to result from strong sampling error due to localized task demand, relatively slow moving workers relative to nest size, and strong spatial variation in task demand. To date, specialization has been primarily interpreted with the response threshold concept, which is focused on intrinsic (typically genotypic) differences between workers. Response threshold variation and sampling error due to spatial effects are not mutually exclusive, however, and this study suggests that both contribute to patterns of task bias at the individual level. While spatial effects are strong enough to explain some documented cases of specialization; they are relatively short term and not explanatory for long term cases of specialization. In general, this study suggests that the spatial layout of tasks and fluctuations in their demand must be explicitly controlled for in studies focused on identifying genotypic specialists.
\end{abstract}

Keywords Apis mellifera - Division of labor . Response threshold concept . Task allocation . Social insects

B. R. Johnson ( $\square)$

Department of Ecology, Behavior, and Evolution,

University of California, San Diego, 9500 Gilman Dr 0116,

La Jolla, CA 92093-0116, USA

e-mail: bjohnson741@hotmail.com

\section{Introduction}

Insect colonies exhibit some of the most sophisticated social organizations in nature. The largest societies consist of thousands to millions of workers and are often characterized by elaborate systems of division of labor (reviewed in Beshers and Fewell, 2001). This social complexity is thought to underlie the great ecological success of these insects (Wilson and Hölldobler, 2005). Within the topic of division of labor, much attention has been paid to the role played by specialists, workers with a bias for a particular task (Visscher, 1983; Moore et al., 1987; Kolmes, 1989; O’Donnell and Jeanne, 1992; Trumbo et al., 1997; Breed et al., 2002; Johnson, 2002; Dornhaus, 2008; Jandt et al., 2009). Removing corpses from a honey bee colony, guarding the nest entrance, and grooming, for example, are tasks performed by a small portion of the colony's workers. Within the subset of workers performing these tasks, some perform them much more often than would be expected by chance alone (Visscher, 1983; Moore et al., 1987; Kolmes, 1989; Trumbo et al., 1997; Breed et al., 2002). Although the honey bee provides the most examples of such behavior, these patterns have been observed in ants and wasps as well and have been central to general models of task allocation (O'Donnell and Jeanne, 1992; Bonabeau et al., 1998; Page and Mitchell, 1998; Cox and Myerscough, 2003; Dornhaus, 2008; Langridge et al., 2008).

Most experimental work on specialization has focused on differences in response threshold as the explanation (Moore et al., 1987; Robinson and Page, 1988; Beshers and Fewell, 2001; Breed et al., 2002). The earliest studies showing bias in task performance gave rise to the hypothesis that workers have different thresholds for the stimulus level that will elicit task performance, such that some workers will respond to a low stimulus for a task 
while others will only respond to a much higher stimulus (Calderone and Page, 1988, 1992; Robinson and Page, 1988; Breed and Rogers, 1991). Workers with the lowest response thresholds are thought to be specialists. A rich body of work has shown that such variation in response threshold does exist (Pankiw and Page, 2000; Beshers and Fewell, 2001; Weidenmuller, 2004; Oldroyd and Thompson, 2007). Further, numerous studies have shown that differences in genotype underlie some of this variation (Breed and Rogers, 1991; Stuart and Page, 1991; Page et al., 1995; O'Donnell, 1998; Ranger and O'Donnell, 1999; Blatrix et al., 2000; Kryger et al., 2000; Jones et al., 2004). Workers within different patrilines in the honey bee, for example, have different probabilities of working on most tasks studied to date (reviewed in Beshers and Fewell, 2001; Oldroyd and Thompson, 2007). Similar patterns have been found in stingless bees, wasps, and ants (Stuart and Page, 1991; O'Donnell, 1998; Blatrix et al., 2000). In addition, a smaller number of studies have shown that response thresholds can vary with experience (Weidenmuller, 2004; Langridge et al., 2008). In other words, workers become more likely to perform a task each time they perform it. In short, there is clear evidence in support of the response threshold model. Theoretical studies of specialization have largely mirrored experimental work by focusing on response threshold variation (reviewed in Beshers and Fewell, 2001) with far fewer models focusing on spatial effects (see Tofts and Franks, 1992; Franks and Tofts, 1994 for exceptions). As several authors have pointed out, however, spatial effects should contribute to most aspects of task allocation, and division of labor, and are therefore a key understudied factor in this field (Sendova-Franks and Franks, 1994, 1995).

The role that spatial effects might play in generating task choice bias is quite intuitive. The honey bee nest has a strong spatial structure with a honey zone at the top, a brood zone in the center, and a dance floor at the bottom (Seeley and Morse, 1976). Rare tasks, such as guarding and undertaking, also occur within localized areas (Visscher, 1983; Moore et al., 1987). Within this spatial context, bees alternate between periods of working, inactivity, and patrolling (Johnson, 2008a, b). Hence, as a worker cycles between activity and inactivity, it has a finite chance of experiencing the same task stimulus repeatedly, by chance alone, due to either returning to same zone or staying within one work-zone between work bouts. The factors that should influence repeated stimulus encounters are the area of the work zone, the availability of work within that zone, and how quickly workers move away from work zones. The purpose of this study is to explore, using individual oriented simulations, whether these factors generate strong (and statistically significant) task choice biases (specialization patterns) in middle age honey bees (the group that has been the focus of the most work on this topic). In so doing, a number of related questions will be addressed, such as are spatial effects strong enough to explain empirical specialization patterns and what sort of sampling methodologies might be useful for controlling for spatial effects and isolating response threshold effects.

\section{Methods}

In previous studies, I showed that workers alternate between three behavior states: inactive, working, and patrolling (Johnson, 2008a, b). These data were used to derive and parameterize an agent based model in the programming language, Netlogo (Wilensky, 1999). A related paper uses this model to explore colony level patterns of task allocation (Johnson, 2009), while the present study focuses on individual-level patterns. The essentials of the model are summarized below, but the reader should see Johnson (2009) for additional details of the modeling procedure and a complete copy of the computer code.

\section{Nest characteristics}

The simulated nest was composed of 528 total patches broken into five work zones (Fig. 1). Each patch corresponds to $2.5 \mathrm{~cm}^{2}$ of actual nest. Only one task is conducted within each work zone. Each patch has a stimulus level for one task that is the amount of work available on that patch. When a bee begins to work on a patch, for example, the stimulus level falls by one. Stimulus level increases by one when a bee quits working on a patch. Stimulus levels for each set of simulations are shown in their respective figure legends. The high stimulus level

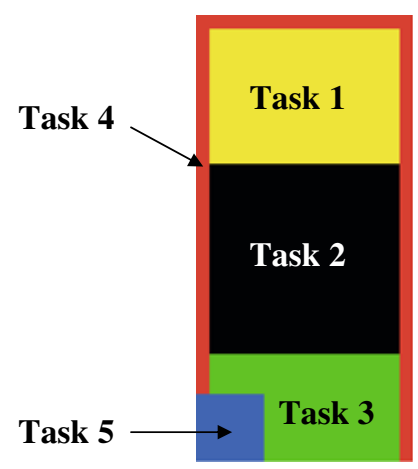

Fig. 1 Image capture of the nest used in all simulations. Each zone is composed of numerous patches, each of which has a stimulus set to the level of work available on that patch. The work zones match the natural distribution of three common and two rare task zones. Zones 1-3 represent from top to bottom, the honey zone, the brood zone and the dance floor. Zone 4 represents the edge of the nest where tasks such as working tree resin occur. Zone 5 represents the area near the nest entrance where guarding and undertaking occur 
used for task 5 in Fig. 5 is based on studies of undertaking specialization in which large numbers of corpses were introduced to colonies (Visscher, 1983, Trumbo et al., 1997).

\section{Worker behavior}

Workers are identical autonomous agents. They do not differ from one another in terms of probability of performing tasks or in movement rate. Workers transition between working, patrolling (a random walk in which they are insensitive to task demand), and inactivity. When working, bees conduct a random search for work, working on the first task they encounter with a stimulus greater than or equal to 1 . Each bees sensing radius is one patch, the patch on which it currently resides. When patrolling, workers conduct a correlated random walk throughout the nest. Inactive workers do not either move or work. Each worker's task choices were recorded hourly starting at hour 3 in each simulation to allow the model to come to come to an equilibrium task allocation pattern.

For simulations lasting longer than 1 day, night time behavior had to be estimated. Middle age bees have been shown to sleep at night (Klein et al., 2008). They are activated in the morning by the foragers who communicate the need for activity with the vibration dance (reviewed in Schneider and Lewis, 2004). Thus, workers should move far slower at night than during the day. For simulations shown in the text, the night time movement rate was $25 \%$ of the day time rate. Setting night time movement rate to the daytime rate (the maximum) does not qualitatively change any of the results.

\section{Identification of specialists}

Some studies assume all the workers observed on a rare task are specialists (Visscher, 1983; Moore et al., 1987). Other studies compare the distribution of individual task performance rates to a Poisson distribution of the same mean and conclude that specialists exist if there are statistical differences between the distributions (Kolmes, 1989; Johnson, 2002). In this study, specialists were identified by comparing the distribution of observed task performance rates to a random distribution of the same mean following the procedure of Kolmes (1989). The first category in which the expected number was less than 0.1 bees was used as the threshold for specialization. The observed value was typically over a hundred times the value of this specialization threshold. The binomial distribution was used, as opposed to the Poisson, because it is more accurate when the number of events is known.

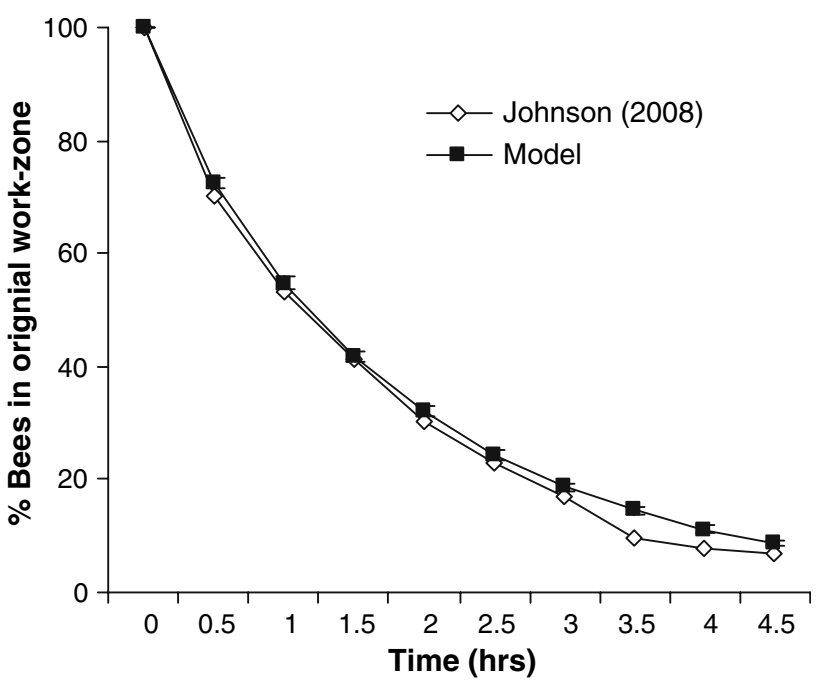

Fig. 2 Validation that the model produces worker movement behavior consistent with real bees. Figure 6a from Johnson (2008a) was modeled to ensure that simulated bees leave their work-zone at the appropriate rate. Bee location was sample every half hour in a simulated nest with the same characteristics as those used in the experimental study

\section{Parameterization}

For all simulations, unless otherwise stated in the text or figure legends, the worker population was 2,500 . Stimulus levels and the number of patches per task, the other important parameters, are given in the figure or table legends. Figure 2 shows that the movement rates (probability of a worker leaving their work zone) of simulated bees matches that of real bees (recorded in Johnson, 2008a). Much further validation and parameterization of the model are reported in Johnson (2009).

\section{Results}

Patterns of task choice at the individual level

Figure 3 shows the individual-level task performance rates (hourly scans for $12 \mathrm{~h}$ ) for middle age bees within the nest depicted in Fig. 1. The distributions for all five tasks differ from random, the prediction if bees have the same probability of performing each task (chi-square test: $N=2,500$ for all comparisons, task $1: \chi_{7}^{2}=2297.49$, $P<0.01$, task $2: \chi_{6}^{2}=1118.37, \quad P<0.01$, task 3 : $\chi_{5}^{2}=1478.41, P<0.01$, task $4: \chi_{4}^{2}=836.83, P<0.01$, task 5: $\left.\chi_{3}^{2}=875.12, P<0.01\right)$. Hence, although the workers in the model are identical (no response threshold variability), there are apparent specialists for each task. Our focus now is to explore the factors that contribute to these biases. Task 5 , which has a spatial distribution (small and 

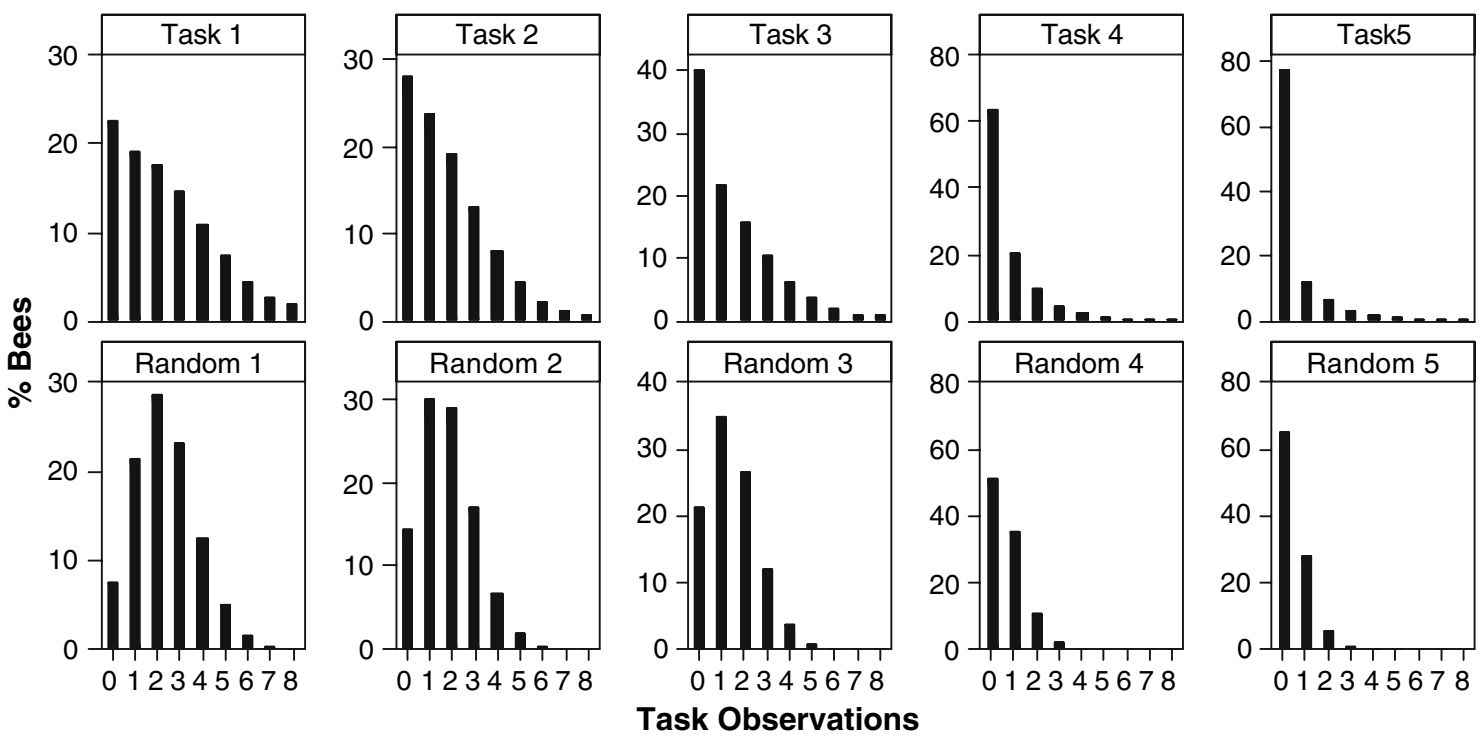

Fig. 3 Number of times bees were observed performing a task over 12 observation periods in the simulated nest shown in Fig. 1 [stimulus $\left.1\left(S_{1}\right)=6, S_{2}=2, S_{3}=4, S_{4}=2, S_{5}=6\right)$. Each distribution is strongly right skewed and statistically different from a random

near the entrance) that matches that of the two tasks for which specialization has been the most studied, undertaking and guarding, will be the focus.

\section{Effect of variation in task area}

Task choice bias should depend on spatial variation in task demand because if a task is only conducted within a limited region, then workers near that region will have some finite probability of experiencing stimuli for it, while workers distant from that region will have no chance of experiencing the relevant task stimuli. We should expect this effect to decrease as the area the task covers increases to the extent that all the workers have an equal chance of experiencing the stimulus. Figure 4 demonstrates this by showing a decrease in the number of apparent specialists as the area a task covers increases.

\section{Effect of variation in task demand}

Individual task performance rates should depend in part on variation in task demand. This is because if work is no longer available in a particular region of the nest, then a worker will move from that zone in the course of searching for work. However, if work is still available in their current location, bees should be more likely to remain there and continue with the same task over multiple bouts of work. Figure 5 supports this hypothesis by showing that as demand for a task increases (holding area constant), the number of workers with a bias towards performing the task increases until a threshold number of specialists is reached.

distribution of the same mean (see text for statistics). Hence, although the bees in the model are identical, there are apparent specialists (bees with strong task choice biases) for all five tasks

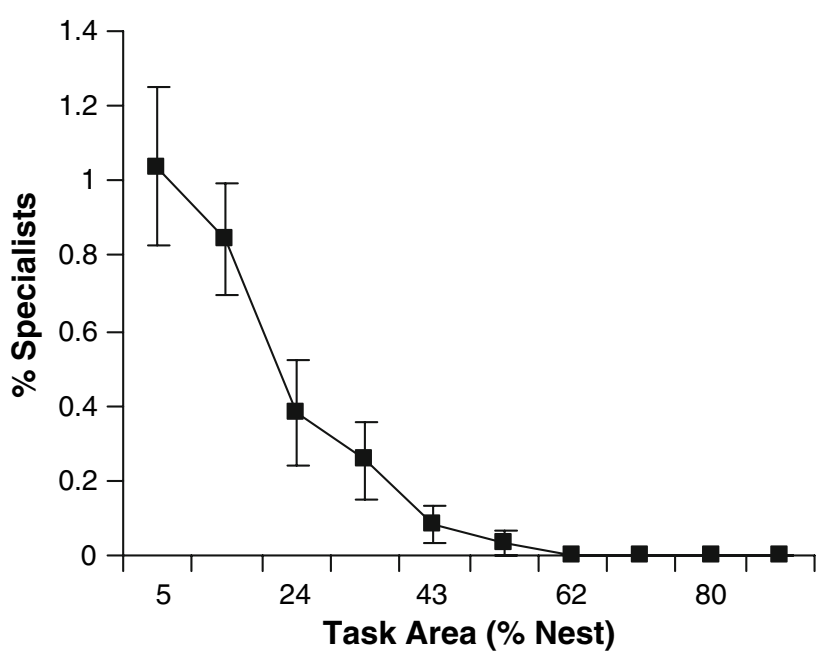

Fig. 4 Results of simulations in which task 5 area is varied. Stimulus level was held constant at 6 per patch for the focal task (number 5); other stimulus levels were as in Fig. 3. As task area increases, the number of apparent specialists decreases to zero. Thus, as area increases, the probability of all workers experiencing the task stimulus at the same rate increases until no workers show a bias in the performance of the task

Essentially, above the threshold there is always work available so further increases have little to no effect.

\section{Comparisons to experimental results}

Three studies were chosen for comparisons between simulations and results. Visscher (1983) found that few bees overall participate in undertaking and, among those that do, a small number engage in the task repeatedly. Johnson 


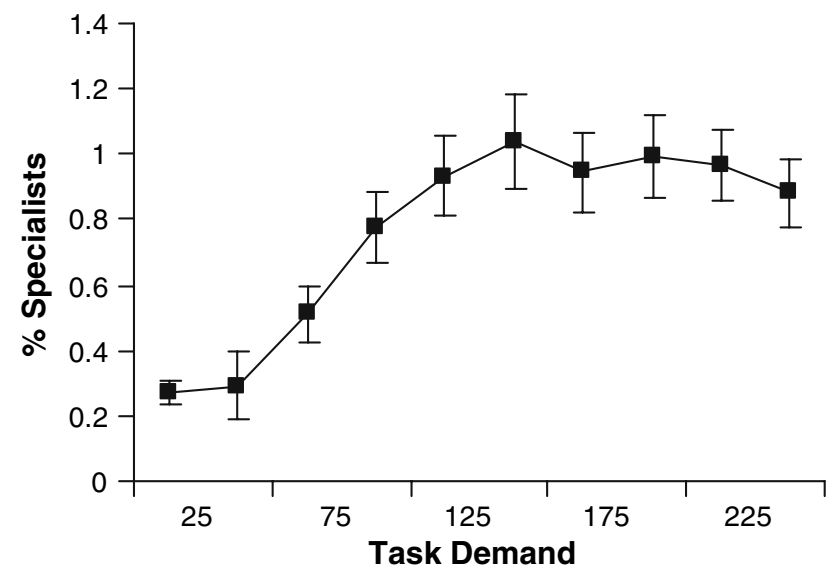

Fig. 5 Results of simulations in which task 5 demand was varied from 25 to 250 ( 25 patches with stimulus ranging from 1 to 10 ). Stimuli levels as in Fig. 2. The number of specialists is an increasing function of task demand

(2002) reported a bias in task performance for the common task of wax working, which occurs over a large area of the nest. Table 1 shows that the degree of bias found in these studies is within the range explainable by the effects explored in this study [chi-square test, Visscher (1983): $\chi_{2}^{2}=0.93, N=92, P=0.63$; Johnson (2002): $\chi_{6}^{2}=5.88$, $N=385, P=0.436]$. These results are useful for showing how strong spatial effects can be given biologically realistic parameter settings (found by parameter tuning), however, it is not claimed that the biases found by these authors are due solely to spatial effects. Moore et al. (1987) and Trumbo et al. (1997) reported long term biases (over many days) in a small minority of bees for the tasks of guarding and undertaking. Table 1 shows that these levels of specialization could not be generated by the factors explored in the present study (chi-square test: $\chi_{3}^{2}=19.96$, $N=1,035, P<0.01)$. The factors explored in the present study, easily lead to biases over several days; however, the percentage of bees with such biases is lower than reported in these studies.

\section{Controlling for spatial effects}

This work has shown that biases for tasks can be generated by spatial effects alone. A key question is how can such effects be controlled for by researchers interested in other mechanisms of generating task bias? As Fig. 4 shows, spatial effects are most important for those tasks that cover a small area. However, even when tasks cover small areas; over a long enough time period, all of the bees might ultimately experience the task at the same rate as they move about the nest. Figure 6 explores this possibility by showing the results of long term simulations on task performance rates. Middle age bees stay within their caste for
Table 1 Comparison of model results with experimental data

\begin{tabular}{|c|c|c|c|}
\hline Undertaking observations & $\begin{array}{l}\text { Empirical } \\
\text { count }\end{array}$ & Model & SD \\
\hline \multicolumn{4}{|l|}{ Visscher (1983) } \\
\hline 1 & 60 & 59.18 & 6.36 \\
\hline 2 & 21 & 24.04 & 3.50 \\
\hline 3 & 6 & 9.18 & 2.78 \\
\hline 4 & 2 & 3.02 & 1.62 \\
\hline 5 & 2 & 1.42 & 1.21 \\
\hline $6+$ & 1 & 0.78 & 0.84 \\
\hline Wax working observations & $\begin{array}{l}\text { Empirical } \\
\text { count }\end{array}$ & Model & SD \\
\hline \multicolumn{4}{|l|}{ Johnson (2002) } \\
\hline 0 & 80 & 89.72 & 7.65 \\
\hline 1 & 90 & 93.72 & 7.81 \\
\hline 2 & 72 & 77.94 & 7.17 \\
\hline 3 & 61 & 56.46 & 6.96 \\
\hline 4 & 40 & 34.68 & 5.08 \\
\hline 5 & 20 & 18.46 & 3.44 \\
\hline $6+$ & 22 & 14.02 & 4.03 \\
\hline Days guarding & $\begin{array}{l}\text { Empirical } \\
\text { count }\end{array}$ & Model & SD \\
\hline \multicolumn{4}{|l|}{ Moore et al. (1987) } \\
\hline 0 & 929.57 & 917.64 & 8.62 \\
\hline 1 & 82.57 & 93.76 & 9.44 \\
\hline 2 & 13.14 & 23.28 & 4.56 \\
\hline 3 & 6.86 & 2.86 & 1.57 \\
\hline 4 & 1.71 & 0.44 & 0.69 \\
\hline 5 & 1.00 & 0.02 & 0.14 \\
\hline 6 & 0.57 & 0 & 0 \\
\hline
\end{tabular}

Visscher (1983): simulation length: $12 \mathrm{~h}$, population size: 1,000; $S_{1}=6, S_{2}=2, S_{3}=4, S_{4}=1, S_{5}=1$, task 5 patches: 18 ; Johnson (2002): population size: 2,$500 ; S_{1}=6, S_{2}=2, S_{3}=4, S_{4}=2$, $S_{5}=6$, task 2 patches: 224 ; length of study: 2 days (6 hourly scans per day). Moore et al. (1987): population size: 1,035; $S_{1}=6, S_{2}=4$, $S_{3}=2, S_{4}=2, S_{5}=1$, task 5 patches: 2 ; length of study 6 days. $12 \mathrm{~h}$ of data collection per day for the first $30 \mathrm{~min}$ of each hour (as per Moore et al. 1987)

approximately 1 week (Johnson, 2008b), making this the maximum biologically relevant length of time (only a tiny percentage of bees show biases over more than 3 or 4 days, however, making this the most realistic length of study). Two tasks, numbers 2 and 5, were the focus. Task 2 covers a large area in the center of the nest and should therefore be encountered often by most bees over a long time period. Figure 6a shows that after 7 days, the distribution for task 2 is nearing the shape of a random distribution; though still significantly different (chi-square test: task 2 : $\chi_{18}^{2}=$ $1198.16, N=2,500, P<0.01)$. Task 5 covers a small area 

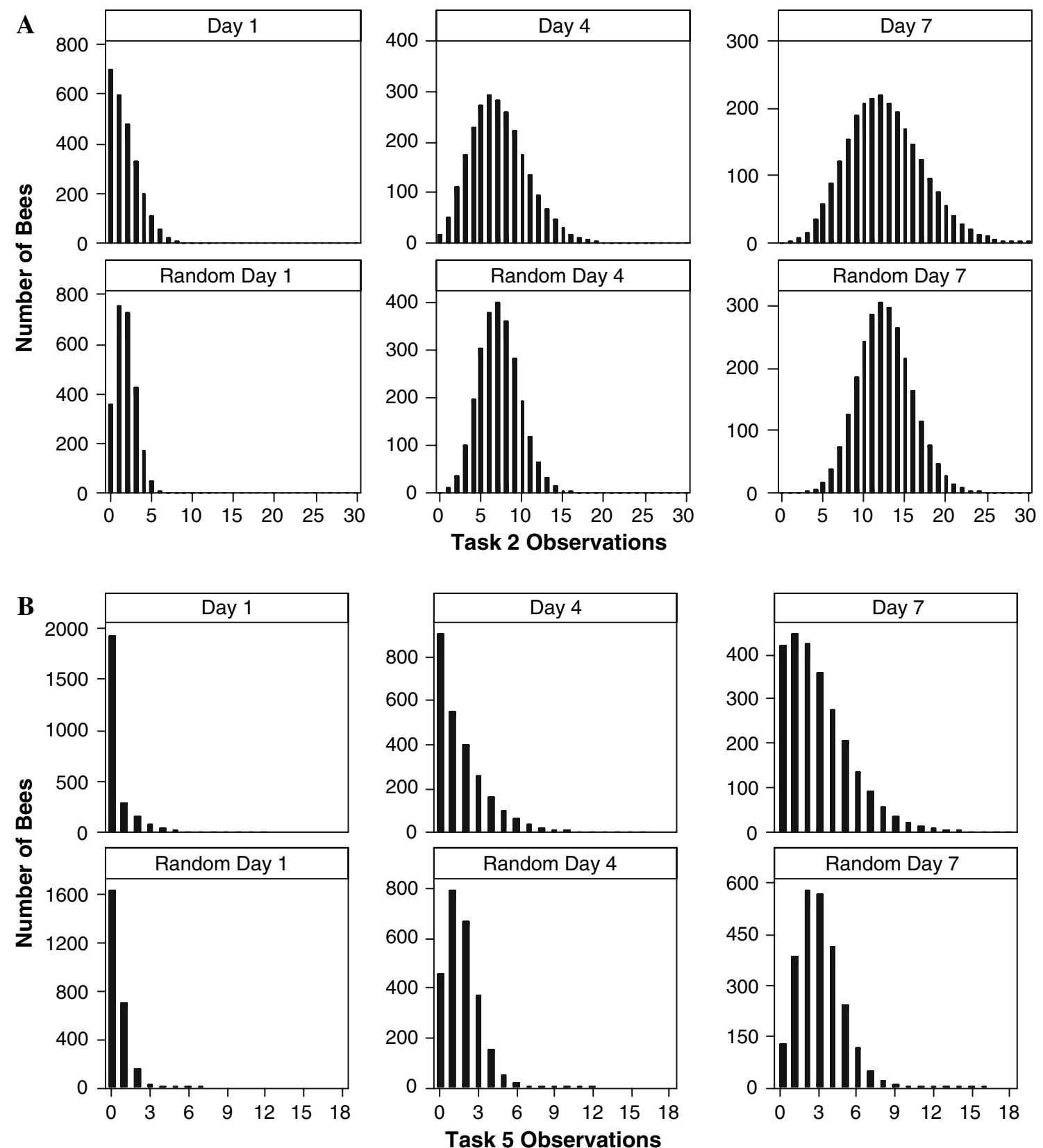

Fig. 6 Results of long term simulations. Stimulus levels as per Fig. 3. Bee behavior was sampled hourly for $12 \mathrm{~h}$ per day for 7 days. Part A shows the task performance distributions of 2,500 bees for task 2 (a common task with a relatively weak bias due to spatial effects). On day 1 , the curve is strongly right skewed, while on day 7 , it

in a corner of the nest and showed the most right skewed distribution after 1 day. Figure $6 \mathrm{~b}$ shows that after 7 days, the curve is still right skewed and strongly different from a random distribution (chi-square test: task 5: $\chi_{9}^{2}=1085.13$, $N=2500, P<0.01)$. In short, spatial effects are strong enough that lengthy observation period alone (with hourly observations) will not control for them. resembles a slightly flattened random distribution (though it is still significantly different from random; see main text for statistics). Part B shows the results for task 5 (a rare task with a strong bias due to spatial effects). The pattern in this case remains strongly right skewed (and different from random) after 7 days (see main text for statistics)

It may also be possible to use infrequent observations to control for spatial effects on task bias, because the longer the bout interval between observations, the farther workers should have moved from their previous location. Figures 7 and 8 explore how well infrequent observations control for spatial effects. Simulations were run with a sampling interval of either 1 or $2 \mathrm{~h}$. One hour interval sampling 

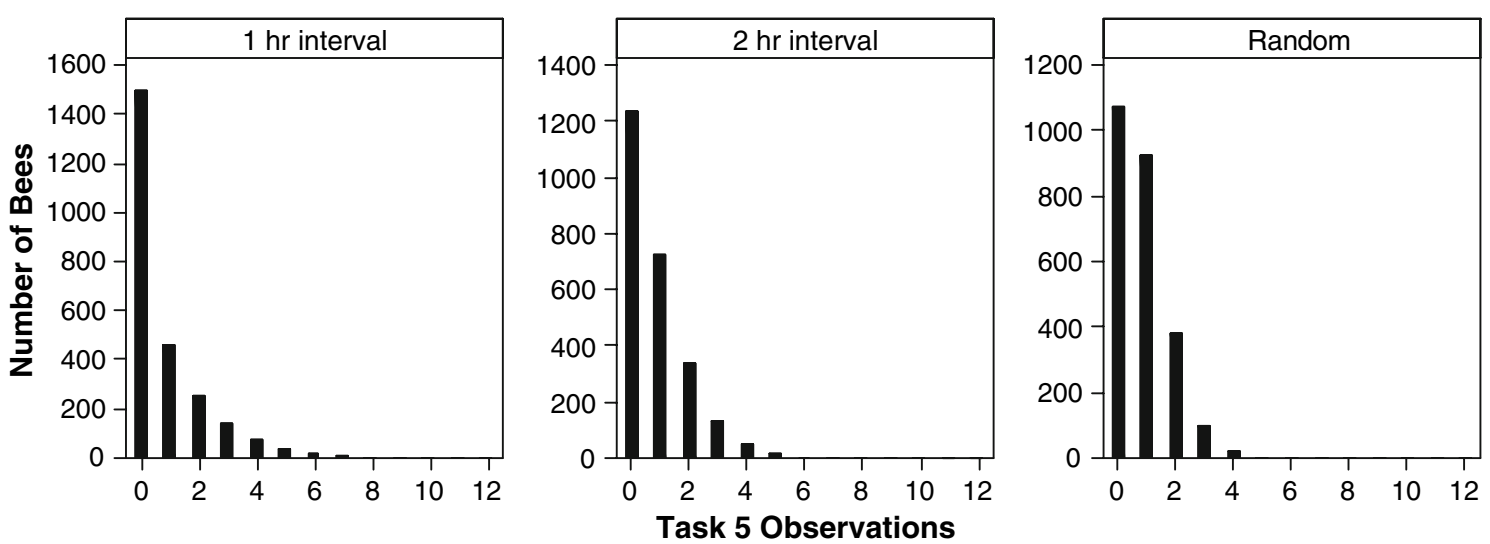

Fig. 7 Results of simulations with infrequent observation intervals. Behavior was sampled every $2 \mathrm{~h}$ for $12 \mathrm{~h}$ for 4 days and every hour for $12 \mathrm{~h}$ for 2 days in two sets of 50 simulations. Thus, quantity of data collected was the same, but the sampling frequency was decreased by $50 \%$ between sets of simulations. Both distributions

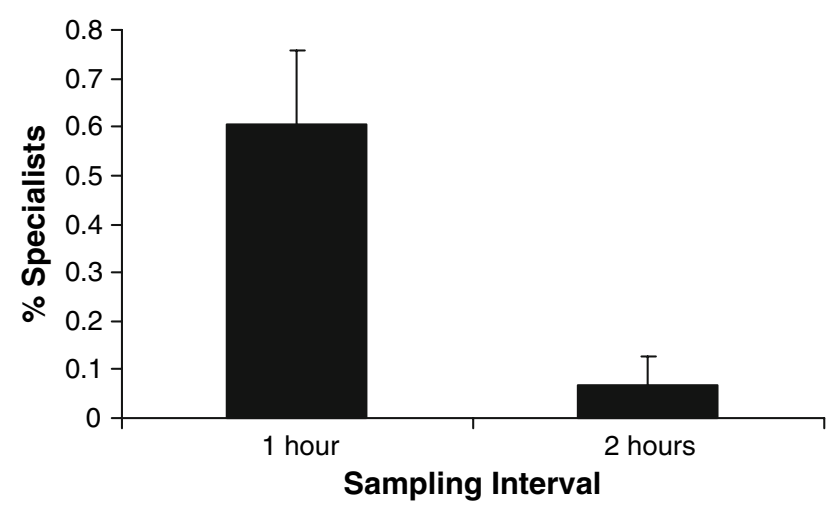

Fig. 8 Results of simulations with infrequent observation intervals. Results are from the same simulations as reported in Fig. 7. The number of specialists is greatly decreased when a more conservative specialist identification statistical procedure is used (statistics in text)

simulations were run for 2 days, while $2 \mathrm{~h}$ interval simulations were run for 4 days. Thus, the amount of data collected was identical, but the sampling interval differed by $50 \%$ between sets of simulations. Although the task performance distributions for task 5 are right skewed in both cases and different from random (chi-square test: $N=2,500$ for both comparisons, $1 \mathrm{~h}$ intervals: $\chi_{4}^{2}=$ 1083.93, $P<0.01 ; 2$ h intervals: $\chi_{4}^{2}=174.94, P<0.01$; Fig. 7), the number of apparent specialists is greatly reduced with $2 \mathrm{~h}$ intervals between observations (number of specialists: $1 \mathrm{~h}$ interval $=0.61 \% \pm 0.15,2 \mathrm{~h}$ inter$\mathrm{val}=0.07 \pm 0.06 \%$; Mann-Whitney test: $W=1276.0$, $N_{1}=50, N_{2}=50, P<0.01$; Fig. 8). Thus, infrequent observations are a possible method for mitigating (though not eliminating) spatial effects on the generation of task bias. differ from random distributions with the same mean (see text for statistics). Infrequent observations thus do not completely control for task bias due to sampling error when the bias in recorded in this fashion

\section{Discussion}

This work shows that spatial effects alone can cause sampling errors strong enough to generate task choice bias at the individual level for both common and rare tasks. Essentially, workers near a localized task zone have a high likelihood of experiencing the relevant task stimulus repeatedly, while workers distant from the zone have little probability of experiencing it. The resulting task performance distribution is many workers with zero observations and many with more than expected by chance alone. This is the pattern that has been documented for specialist tasks in the honey bee and other species (Visscher, 1983; Moore et al., 1987; Johnson, 2002; Breed et al., 2002). However, there is also strong evidence in favor of genotypic variability contributing to these patterns by creating a diversity of response thresholds among workers. Further, as the comparison to experimental data showed, some cases of specialization are too strong to be explained by spatial effects alone. In general, spatial effects and the various forms of response threshold variability are not mutually exclusive and both should contribute to the formation of non-random task performance distributions.

Although specialist workers have received a great deal of attention, it is important to keep in mind the scope of these effects. First, few bees exhibit task choice bias. Experimental work has found that specialists make up 1$2 \%$ of the bees within a nest (Visscher, 1983; Moore et al., 1987; Trumbo et al., 1997; Johnson, 2002). Hence, the degree of specialization generated by the factors explored in the present study (approximately 1\% of the bees were shown to have task choice bias) represents a large fraction of the empirical number. Second, experimental work has shown that not every colony has bees that can be labeled 
specialists. Moore et al. (1987), for example, in their study of guard bees found that out of seven colonies, one colony had no bees that guarded for more than 2 days. Likewise, Breed et al. (2002) in a study of eight colonies had one with an estimated mean of 2980.6 undertakers (a result consistent over five replicates with that colony). In other words, undertaking was not performed by specialists in that colony. It is difficult to explain these patterns with response threshold variation alone (as these effects should occur in every colony), however, the factors explored in the present study shed light on these patterns. Low task demand, higher than normal area of task performance, particularly high density of bees (which leads to difficulty in finding tasks), and so forth are all shown to affect the probability of bees exhibiting task choice bias. As colonies vary strongly in these factors (even within the same environment), it is not surprising that large variation exists in the prevalence of specialization (task choice bias) between colonies.

The results of the present study, along with previous work, suggest a hypothesis that could explain most of the biases seen to date for honey bees. Perhaps the most specialized bees are groomers (Kolmes, 1989). These specialists have the highest rate of task performance and, most important, their specialized task occurs with equal frequency all over the nest. Thus, spatial effects cannot give rise to this pattern. Genotypic variability in response threshold is a more likely explanation. However, as there should be response threshold variability for every task, it begs the question why there is such strong variability in degree of specialization between tasks such as grooming and undertaking. The present study may shed light on this. The key factor may be how often workers encounter particular task stimuli. For some tasks, such as grooming, which has a global distribution, the probability is 1 , while for other tasks the odds are much less and depend on task area, demand, and so forth. Thus, an integrating hypothesis might be that response threshold variation contributes to task choice over a small spatial scale (within a bee's local environment); however, movement rates and the spatial distribution of tasks define the probability of workers being exposed to task stimuli environments. Hence, tasks that occur in a small area would have strong spatial effects on task bias and relatively lower biases due to variation in response threshold, because workers would only rarely experience the task stimulus. In contrast, tasks that occur over large areas would have weak spatial effects, but strong response threshold variability effects on task bias, because workers are always exposed to the stimulus for the task when searching for work.

Finally, some discussion is necessary to put this study into perspective relative to other theoretical work on related topics. In particular, much theoretical work has been published on the generation of division of labor by intrinsic differences between workers related to differences in response thresholds (Bonabeau et al., 1996; Theraulaz et al., 1998; Gautrais et al., 2002; Merkle and Middendorf, 2004; Jeanson et al., 2007, 2008; Gove et al., 2009). Further, a few models, and now some experimental work, are beginning to look at spatial effects on division of labor (Tofts and Franks, 1992; Franks and Tofts, 1994; Jandt and Dornhaus, 2009; Schmickl and Crailsheim, 2007, 2008). How the present model relates to work on spatial effects and division of labor is covered in (Johnson, 2009). Here, I will focus on how it relates to work on response thresholds. First, it is necessary to point out the fundamental difference between caste based division of labor, in which workers have a limited, but still extensive, task repertoire, and specialization, in which a small number of individuals have a strong bias for one task. The present study focuses on specialization only. Second, the present study shows how apparent specialization can emerge from identical workers in a spatially complex environment. This is important for how one designs and interprets studies on specialization. As I argue above with respect to the integrating hypothesis for spatial effects and genotypic effects on generation of task bias, it also has relevance for studies on response threshold variation. In general, with respect to previous modeling work on response thresholds, the conclusion of the present study is that all such effects should be explored again in a context in which there is strong spatial heterogeneity of tasks. While it is unlikely that the basic conclusions of these studies would require changing, it is likely that interesting interactions will emerge from the effects of fixed and variable response threshold effects in a spatially complex environment.

Acknowledgments I thank Neil Tsutsui and two anonymous referees for careful reading of the manuscript. The research reported here complies with the laws of the United States of America. This work was supported by a National Science Foundation Postdoctoral Fellowship.

Open Access This article is distributed under the terms of the Creative Commons Attribution Noncommercial License which permits any noncommercial use, distribution, and reproduction in any medium, provided the original author(s) and source are credited.

\section{References}

Beshers S.N. and Fewell J.H. 2001. Models of division of labor in social insects. Annu. Rev. Entomol. 46: 413-440

Blatrix R., Durand J.L. and Jaisson P. 2000. Task allocation depends on matriline in the ponerine ant Gnamptogenys striatula Mayr. J. Insect Behav. 13: 553-562

Bonabeau E., Theraulaz G. and Deneubourg J.-L. 1996. Quantitative study of the fixed threshold model for the regulation of division of labour in insect societies. Proc. R. Soc. Lond. B. 263: 1565-1569 
Bonabeau E., Theraulaz G. and Deneubourg J.-L. 1998. Fixed response thresholds and the regulation of division of labor in insect societies. Bull. Math. Biol., 60: 753-807

Breed M.D. and Rogers K.B. 1991. The behavioral genetics of colony defense in honeybees: variability for guarding behavior. Behav. Gen. 21: 295-303

Breed M.D., Williams D.B. and Queral A. 2002. Demand for task performance and workforce replacement: Undertakers in honeybee, Apis mellifera, colonies. J. Insect Behav. 15: 319-329

Calderone N.W. and Page R.E. 1988. Genotypic variability in age polyethism and task specialization in the honey bee, Apis mellifera (Hymenoptera, Apidae). Behav. Ecol. Sociobiol. 22: 17-25

Calderone N.W. and Page R.E. 1992. Effects of interactions among genotypically diverse nestmates on task specialization by foraging honey bees (Apis mellifera). Behav. Ecol. Sociobiol. 30: $219-226$

Cox M.D. and Myerscough M.R. 2003. A flexible model of foraging by a honey bee colony: the effects of individual behavior on foraging success. J. Theor. Biol. 223: 179-197

Dornhaus A. 2008. Specialization does not predict individual efficiency in an ant. PLOS Biol. 6: 2368-2375

Franks N.R. and Tofts C. 1994. Foraging for work: how tasks allocate workers. Anim. Behav. 48: 470-472

Gautrais J., Theraulaz G., Deneubourg J.-L. and Anderson C. 2002. Emergent polyethism as a consequence of increased colony size in insect societies. J. Theor. Biol. 215: 363-373

Gove R., Hayworth M., Chhetri M. and Rueppell O. 2009. Division of labour and social insect colony performance in relation to task and mating number under two alternative response threshold models. Insect. Soc. 56: 319-331

Jandt J. M. and Dornhaus A. 2009. Spatial organization and division of labor in the bumble bee, Bombus impatiens. Anim. Behav. 77: 641-651

Jandt J.M., Huang E. and Dornhaus A. 2009. Weak specialization of workers inside a bumble bee (Bombus impatiens) nest. Behav. Ecol. Sociobiol. 63: 1829-1836

Jeanson R., Clark R.M., Holbrook C.T., Bertram S.M., Fewell J.H. and Kukuk P.F. 2008. Division of labour and socially induced changes in response thresholds in associations of solitary halictine bees. Anim. Behav. 76: 593-602

Jeanson R., Fewell J.H., Gorelick R. and Bertram S.M. 2007. Emergence of increased division of labor as a function of group size. Behav. Ecol. Sociobiol. 62: 289-298

Johnson B.R. 2002. Reallocation of labor in honeybee colonies during heat stress: the relative roles of task switching and the activation of reserve labor. Behav. Ecol. Sociobiol. 51: 188-196

Johnson B.R. 2008a. Global information sampling in the honey bee. Naturwissenschaften 95: 523-530

Johnson B.R. 2008b. Within-nest temporal polyethism in the honey bee. Behav. Ecol. Sociobiol. 62: 777-785

Johnson B.R. 2009. A self-organizing model for task allocation via frequent task quitting and random walks in the honey bee. Am. Nat. 174: $537-547$

Jones J.C., Myerscough M.R., Graham S. and Oldroyd B.P. 2004. Honey bee nest thermoregulation: Diversity promotes stability. Science 305: 402-404

Klein B.A., Olzsowy K.M., Klein A., Saunders K.M. and Seeley T.D. 2008. Caste-dependent sleep of worker honey bees. J. Exp. Biol. 211: $3028-3040$

Kolmes S.A. 1989. Grooming specialists among worker honey bee, Apis mellifera. Anim. Behav. 37: 1048-1049

Kryger P., Kryger U. and Moritz R.F.A. 2000. Genotypical variability for the tasks of water collecting and scenting in a honey bee colony. Ethology 106: 769-779
Langridge E.A., Sendova-Franks A.B. and Franks N.R. 2008. How experienced individuals contribute to an improvement in collective performance in ants. Behav. Ecol. Sociobiol. 62: 447-456

Merkle D. and Middendorf M. 2004. Dynamic polyethism and competition for tasks in threshold reinforcement models of social insects. Adapt. Behav. 12: 251-262

Moore A.J., Breed M.D. and Moor M.J. 1987. The guard honey bee: ontogeny and behavioral variability of workers performing a specialized task. Anim. Behav. 35: 1159-1167

O'Donnell S. 1998. Genetic effects on task performance, but not on age polyethism, in a swarm-founding eusocial wasp. Anim. Behav. 55: 417-426

O'Donnell S. and Jeanne R.L. 1992. Lifelong patterns of forager behavior in a tropical swarm-founding wasp: effects of specialization and activity level on longevity. Anim. Behav. 44: 10211027

Oldroyd B.P. and Thompson G.J. 2007. Behavioral genetics of the honey bee, Apis mellifera. Adv. Insect Physiol. 33: 1-49

Page R.E. and Mitchell S.R. 1998. Self organization and the evolution of division of labor. Apidologie 29: 101-120

Page R.E., Robinson G.E., Fondrk M.K. and Nasr M.E. 1995. Effects of worker genotypic diversity on honey bee colony development and behavior (Apis mellifera L). Behav. Ecol. Sociobiol. 36: 387396

Pankiw T. and Page R.E. 2000. Response thresholds to sucrose predict foraging division of labor in honeybees. Behav. Ecol. Sociobiol. 47: 265-267

Ranger S. and O’Donnell S. 1999. Genotypic effects on forager behavior in the neotropical stingless bee Partamona bilineata (Hymenoptera : Meliponidae). Naturwissenschaften 86: 187-190

Robinson G.E. and Page R.E. 1988. Genetic determination of guarding and undertaking in honeybee colonies. Nature 333: 356-358

Schmickl T. and Crailsheim K. 2007. HoPoMo: A model of honeybee intracolonial population dynamics and resource management. Ecol. Model. 204: 219-245

Schmickl T. and Crailsheim K. 2008. TaskSelSim: a model of the self-organization of the division of labour in honeybees. Math. Comp. Model. Dyn. 14: 101-125

Seeley T.D. and Morse R.A. 1976. The nest of the honey bee (Apis mellifera). Insect. Soc. 23: 495-512

Sendova-Franks A.B. and Franks N.R. 1994. Social resilience in individual worker ants and its role in division of labor. Proc. $R$. Soc. Lond. B. 256: 305-309

Sendova-Franks A.B. and Franks N.R. 1995. Spatial relationships within nests of the ant Leptothorax unifasciatus (Latr.) and their implications for their division of labor. Anim. Behav. 50:121-136

Schneider S.S. and Lewis L.A. 2004. The vibration signal, modulatory communication and the organization of labor in honey bees, Apis mellifera. Apidologie 35: 117-131

Stuart R.J. and Page R.E. 1991. Genetic component to division of labor among workers of a leptothoracine ant. Naturwissenschaften 78: $375-377$

Theraulaz G., Bonabeau E., Deneubourg J.-L. 1998. Response threshold reinforcement and division of labour in insect societies. Proc. R. Soc. Lond. B. 265: 327-332

Tofts C. and Franks N.R. 1992. Doing the right thing: ants, honeybees, and naked mole-rats. Trends Ecol. Evol. 7: 346-349

Trumbo S.T., Huang Z.Y. and Robinson G.E. 1997. Division of labor between undertaker specialists and other middle-aged workers in honey bee colonies. Behav. Ecol. Sociobiol. 41: 151-163

Visscher P.K. 1983. The honey bee way of death: necrotic behavior in Apis mellifera colonies. Anim. Behav. 31: 1070-1076 
Weidenmuller A. 2004. The control of nest climate in bumblebee (Bombus terrestris) colonies: interindividual variability and self reinforcement in fanning response. Behav. Ecol. 15: 120-128

Wilensky U. 1999. NetLogo. http://ccl.northwestern.edu/netlogo/. Center for Connected Learning and Computer-Based Modelling, Northwestern University. Evanston, IL
Wilson E.O. and Hölldobler B. 2005. The rise of the ants: A phylogenetic and ecological explanation. Proc. Natl. Acad. Sci. USA 102: 7411-7414 